(C) 1999 IEEE. Personal use of this material is permitted. Permission from IEEE must be obtained for all other uses, in any current or future media, including reprinting/republishing this material for advertising or promotional purposes, creating new collective works, for resale or redistribution to servers or lists, or reuse of any copyrighted component of this work in other works. 


\title{
An Inexpensive Phantom for Evaluating Gated Blood Pool Data Acquisition/Processing Systems at Heart Rates Above 400/min
}

\author{
J.J. Vaquero, S. Siegel ${ }^{1}$, J. Seidel, M.V. Green \\ Nuclear Medicine Department \\ National Institutes of Health, Bethesda MD 20892
}

\begin{abstract}
Equilibrium gated blood pool imaging of the heart is a common diagnostic procedure for visualizing cardiac function in human subjects. Recently, this procedure has been modified to evaluate cardiac function in mice. However, the high heart rates encountered in these animals (often greater than 400 beats/min) can confound R-wave trigger devices, acquisition systems and image processing software containing default conditions tailored specifically to the lower heart rates of human subjects. In order to determine whether data acquisition and processing components of a commercial or self-generated gated blood pool imaging procedure are performing properly, input of known timing and imaging signals that mimic those generated during high heart rate gated blood pool imaging is required. Here, we describe an inexpensive phantom that is suitable for initial evaluation of an unknown system or for ongoing QC of a previously verified system.
\end{abstract}

\section{INTRODUCTION}

In order to verify the accuracy of an imaging procedure, it is customary to construct and image a "phantom" that physically mimics conditions likely to be encountered during that procedure. For small animal gated blood pool imaging, such a phantom must generate timing signals at a rate that mimics the frequency of occurrence of R-waves in the animals' EKG signal. At the same time, the phantom must also produce a spatially varying radiation pattern that changes during a cycle. Although both of these conditions have been achieved with elaborate time-varying cardiac phantoms that closely mimic the beating human heart, it is also possible to meet these conditions with a simple phantom that simulates only the essential aspects of this dynamic process. In this report, we describe this device and illustrate how it is used to verify the accuracy of amplitude, phase and ejection fraction calculations determined with an experimental imaging system.

\section{MATERIALS AND METHODS}

\section{A. Phantom}

The phantom is a rotating plastic disc that is divided into upper and lower compartment as shown in Figure 1 . The lower compartment is a milled annular chamber of constant depth but with a width that varies sinusoidally around the disc.

1 Current address: Concorde Microsystems,

Cogdill Rd, Suite 500, Knoxville, Tennessee 37932
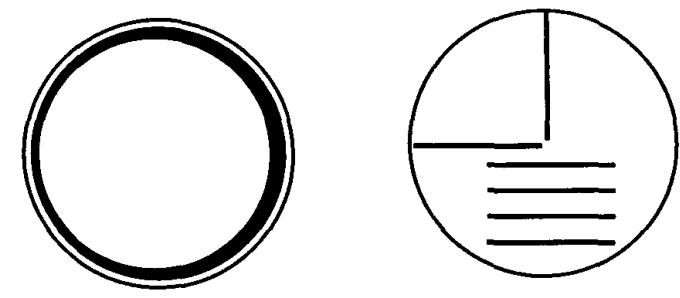

lower compartment

upper compartment

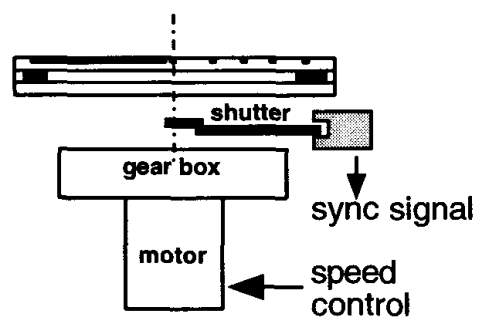

Figure 1. Layout of the sources disk and side view of the complete assembly.

The minimum width is half the maximum width. The upper compartment consists of a series of shallow, milled slots into which capillary tubes can be placed.

Two of these slots are oriented radially and four tangentially. In use, several capillary tubes are filled with a uniform solution of $\mathrm{Tc}-99 \mathrm{~m}$ in water and taped in place in the slots. When the annular chamber is used, a uniform solution of Tc-99m is injected through one of two filling ports until the chamber is completely full and free of air bubbles. The filling and exhaust ports are sealed with metal screws.

As shown in Figure 1, the disc is connected to a shaft rotated by a geared-down, variable speed motor. A thin metal plate or shutter attached to this shaft interrupts a light beam falling on a photocell once every rotation. The photocell signal, in turn, generates a sync pulse that marks the onset of the next cycle. In use, the disc is loaded with the appropriate sources and the whole device placed within the field-of-view of the imaging system. The sync pulse line is connected to the acquisition system, the motor set to the desired rotational speed and imaging initiated. Parameters extracted from the acquired images can then be compared with values calculated from the known phantom geometry. 
We used this phantom to verify the accuracy of amplitude, phase and volume variations calculated from data acquired by a gamma camera equipped with parallel hole collimation.

\section{B. Data Collection}

A single capillary tube filled with $\mathrm{Tc}-99 \mathrm{~m}$ solution was taped into one of the radial slots. The disc was then set rotating at 411 cycles per minute and gated imaging begun. The gated image sequences created from these data collections are shown in Figure 2.
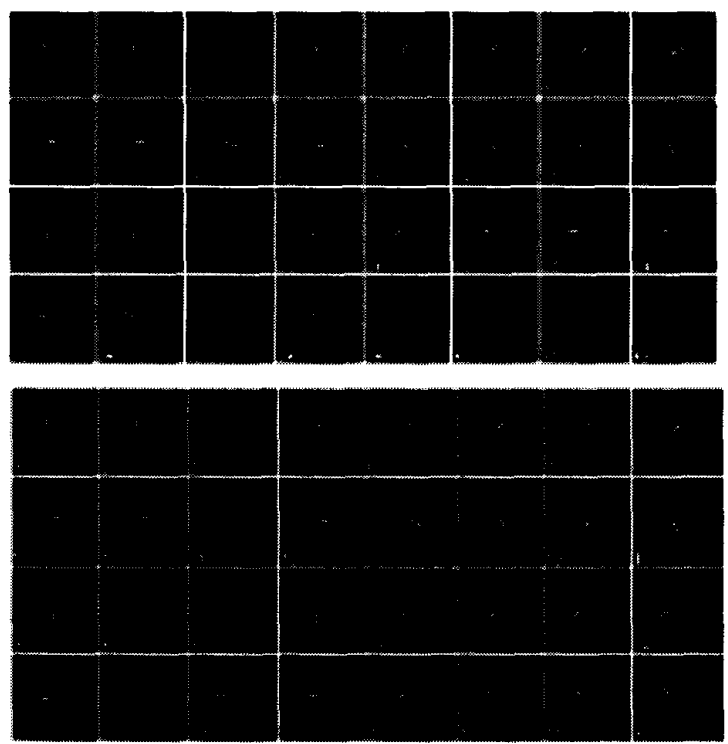

Figure 2. Gated image sequences of a single rotating capillary tube framed with $1 \mathrm{~ms}$ (top) and $0.1 \mathrm{~ms}$ (bottom) time resolution. Note the differences between studies in frames 29 to 32 .

Upon completion of this acquisition, the capillary tube was removed and the annular chamber filled with Tc-99m solution. The disc was again set in motion at the same speed and data acquisition initiated. After completion of this study, the motor speed was increased to 608 cycles per minute and the phantom imaged again.

\section{Data Analysis}

The rotating line source image data were analyzed with supplied software to produce amplitude and phase images (Figures $3 \mathrm{~A}$ and $\mathrm{B}$, respectively). The result of fitting a $1 / \mathrm{R}$ function to a profile through the amplitude image is shown in Figure 3C. A frequency distribution function of phase values is shown in Figure 3D.

"End diastolic" and "end systolic" gated images of the rotating annular compartment are shown in Figures $4 \mathrm{~A}$ and $\mathrm{B}$, respectively, along with the corresponding amplitude (4C) and phase image (4D). If a narrow ROI is drawn radially outward across the annular pattern, the count rate within that ROI should vary sinusoidally with time. Plots of count rate vs. time during the cycle for the two rotation rates studied are shown in Figures $4 \mathrm{E}$ and $\mathrm{F}$.

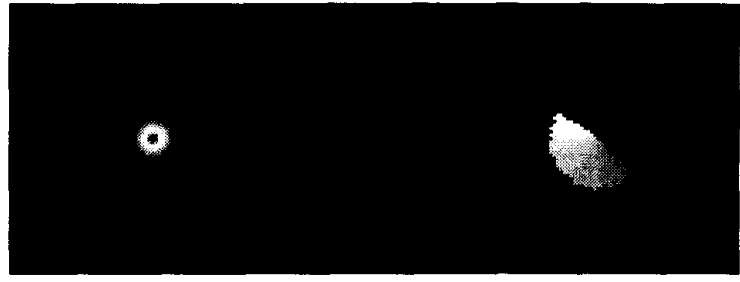

A

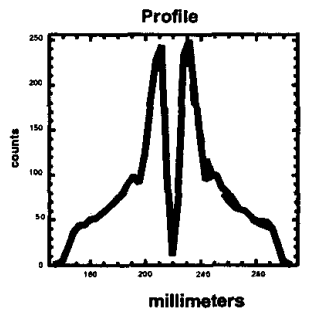

C
B

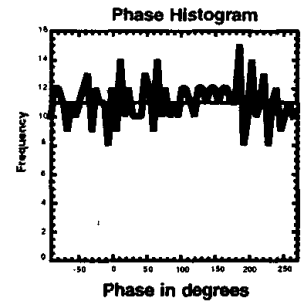

D
Figure 3. Amplitude (A) and phase (B) images computed from the single capillary tube image sequence; profile across $A(C)$, and histogram of B (D).

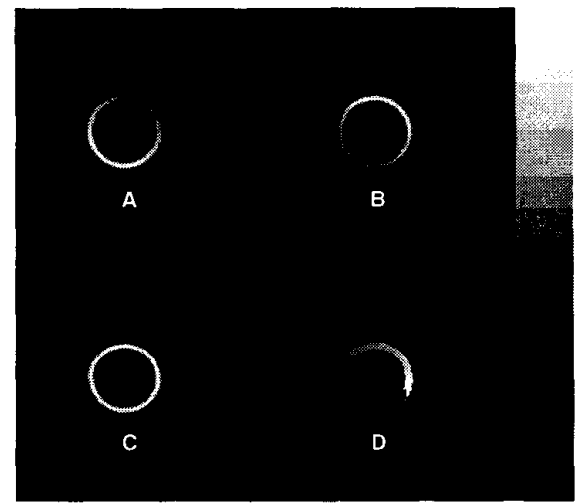

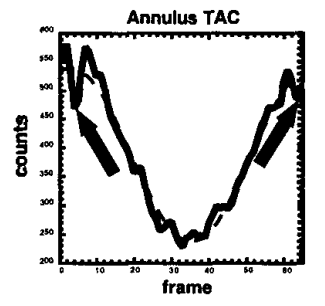

E

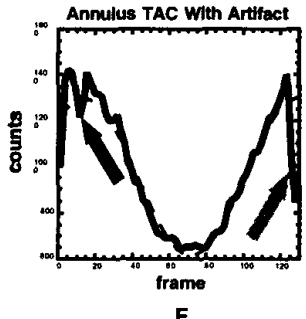

Figure 4. Systole (A), diastole (B), amplitude (C) and phase (D) images computed from the rotating annulus image sequence; normal TAC curve (E), and TAC with artifact (F). Note that "ejection fraction" is $50 \%$ in both.

\section{RESULTS AND DISCUSSION}

\section{A. Rotating Line}

It is not difficult to prove that a rotating line source will give rise to an amplitude image in which radial brightness declines as $1 / \mathrm{R}$ from the center of rotation. This functional form is, in fact, observed when a profile across a diameter of the amplitude image is fit with a $1 / \mathrm{R}$ curve (Figure $3 \mathrm{C}$ ). In addition, it is also easily demonstrated that the phase image of 
a rotating line should be uniformly bright along a given radius, but should increase continuously in brightness with increasing angle around the center. Inspection of Figure 3B indicates that this condition is also met.

Additionally, the number of picture elements per unit phase should be constant so that the frequency distribution of phase values in Figure 3B should be flat. Figure $4 \mathrm{D}$ indicates that this is approximately true, but it is also evident that the sizes of the amplitude and phase images are not the same, though they should be. It would appear, therefore, that phase values are portrayed to the observer only if the amplitude at the same point exceeds some threshold value. The apparent variation in the phase frequency distribution is due, in part, to the fact that at least half of the phase image points have been removed by this condition.

\section{B. Rotating Annulus}

For any gated image of the annulus there should exist another image one-half cycle away in time that is the inverse of the first image. That is, if a point contains the maximum count density in one image, one half cycle later that same point will contain the minimum count density. Inspection of Figure $4 \mathrm{~A}$ and $\mathrm{B}$ indicate that this condition is met.

As with the rotating line, the amplitude image (4C) is uniform, as it must be, and the phase image (4D) has the anticipated radial and angular brightness variations. The fact that this image is about the same size as the amplitude image suggests that the cutoff threshold is calculated as a fraction of the maximum counts in the amplitude image. The rotating annulus has the same amplitude everywhere, whereas the rotating line has a wide variation in brightness from center to edge. The same fractional cutoff that removed phase points from the rotating line phase image would be expected to leave the annulus phase image unchanged since almost all amplitude values would be above the threshold.

The time variations of counts within a narrow, radial ROI overlaying the annulus at two different rotation speeds are shown in Figures $4 \mathrm{E}$ and $\mathrm{F}$. The "ejection fraction" computed from these curves is, in both cases, $50 \%$ as it should be. The arrows in Figure 4E correspond to the time that the two metal filling screws cross the ROI and reduce the counts slightly at these times. Figure 4F shows one of the filling screws (black arrow) and also portrays a frame duration error (grey arrow) that causes counts in the last (and first) image frames to be less than they should be. The source of this error is at present unknown.

\section{CONCLUSION}

Although simple in concept and design, the rotating disc phantom allows a variety of parameters normally computed from gated blood pool image data to be verified at heart rates much higher than normally encountered in human studies. In our evaluation of this particular system, it was found that most of the computed images, e.g. phase, amplitude, etc. were in agreement with predictions based on a mathematical analysis of the rotating phantom.

However, a software threshold was also discovered in addition to a variable framing error that alters the number of events detected at the beginning and end of the cycle. Collectively, these results suggest that this simple and inexpensive phantom can aid the evaluation of gated blood pool imaging/analysis systems operated at high heart rates.

\section{ACKNOWLEDGMENTS}

The authors thank James V. Sullivan and Paul Fitze from the Scientific Equipment \& Instrumentation Branch (DIRSORS-NIH) for their expert assistance in preparing the mechanical components of the phantom. J.J. Vaquero is supported, in part, by a grant from CICYT (Spanish Government). S. Siegel was supported by a grant from the National Research Council.

\section{REFERENCES}

[1] S. L. Bacharach, M. V. Green, and J. S. Borer, "Instrumentation and Data Processing in Cardiovascular Nuclear Medicine," Seminars in Nuclear Medicine, vol. 9, pp. 257-274, 1979.

[2] C. J. Hartley, S. Madala, J. L. Lacy, A. K. Reddy, G. Taffet, E., K. Kurrelmeyer, J. Pocius, T. Pham, N. Nayak, M. L. Entman, and L. H. Michael, "Noninvasive Methods to Measure Cardiovascular Function in Mice," presented at the NIH Bioengineering Symposium, Bethesda, Maryland, 1998.

[3] G. J. Klein, B. W. Reutter, M. H. Ho, J. H. Reed, and R. H. Huesman, "Real-Time System for Respiratory-Cardiac Gating in Positron Tomography," IEEE Transactions on Nuclear Science, vol. 45, pp. 2139-2143, 1998. 